KYUSHU-HET-106

\title{
Probing flavor structure in unified theory with scalar spectroscopy
}

\author{
Kenzo Inoue, Kentaro Kojima, and Koichi Yoshioka \\ Department of Physics, Kyushu University, Fukuoka 812-8581, Japan
}

(March, 2007)

\begin{abstract}
The flavor structure in unified theory is probed with superparticle mass spectrum observed in future particle experiments. A key ingredient is the generation dependence of scalar mass non-degeneracy. The observed non-degeneracy in low-energy regime is shown to provide a direct imprint of flavor structure in high-energy fundamental theory. The implication from flavor-violating rare process is also discussed.
\end{abstract}




\section{Introduction}

Supersymmetry is one of the most prominent solution to the hierarchy problem. A direct consequence of supersymmetry is the existence of superpartners of the standard model (SM) particles. In addition, supersymmetry is strongly favored in view of grand unified theory (GUT). The minimal supersymmetric extension of the SM (MSSM) with TeV-scale superparticles leads to precise unification of the SM gauge couplings at a single scale $\sim 10^{16} \mathrm{GeV}[1]$. Thus supersymmetric GUT is well motivated and has been widely investigated.

The mass spectrum of superparticles, that is soft supersymmetry-breaking parameters, is determined by supersymmetry-breaking dynamics and mediation mechanism [2]. If the mediation scale is higher than the unification scale, e.g. a mediation by Planck scale physics, the superparticle mass spectrum is expected to have some imprints of fundamental theory. This implies that high-energy physics can be probed through the observed superparticle spectrum in low-energy regime. Along this line, possible implications from physics around the GUT scale have been investigated in the literature [3-5].

In this paper we focus on the matter generation structure in unified theory and show that it can be probed with superparticle mass spectrum, once observed in near future particle experiments. In GUT models, quarks and leptons are generally unified into GUT gauge multiplets. It is thus an interesting subject to study the recently revealed disparate flavor structure of quarks and leptons.

The MSSM scalar mass spectrum somehow inherits the matter flavor structure in highenergy theory. In particular, the first and second generation scalars generally have nondegenerate masses even when the universal supersymmetry breaking is assumed at a highenergy scale, as we will discuss below. We point out that particular combinations of MSSM scalar masses are useful for directly extracting the matter flavor structure. With these combinations, the origin of generation structure in unified theory is probed through scalar mass spectrum. We also discuss the implications and constraints on non-universal flavor origins in the lepton sector.

The paper is organized as follows. In Section 2 the generation dependent angles are introduced to describe the flavor dependence of matter structure in unified theory. We examine the resultant non-universality of scalar mass spectrum. Several scalar mass relations for extracting these angles are presented. In Section [3, we show with these relations that the

flavor structure is probed from the scalar mass spectrum, which is expected to be observed in near future particle experiments. Physical implications to flavor origins are discussed in Section 4. Finally we summarize the discussion in Section 5 . 


\section{Matter embedding into unified theory}

\subsection{Generation redundancy}

In grand unified models, the SM matter multiplets are unified into larger GUT gauge multiplets. In the minimal $S U(5)$ model [6], the electroweak doublet quarks $Q_{i}$, up-type quarks $\bar{u}_{i}$ and charged leptons $\bar{e}_{i}$ are unified into decuplets, and the doublet leptons $L_{i}$ and down-type quarks $\bar{d}_{i}$ are combined in anti quintuplets ( $i=1,2,3$ denotes the generations). Further in models with $S O(10)$ or larger unified symmetry, all matter multiplets in one generation are unified into a single gauge multiplet. The simple and naive assumption of matter unification is that three-generation matter multiplets are embedded into GUT multiplets in a completely parallel fashion.

While the unification of MSSM gauge couplings suggests the existence of grand unification at high-energy regime, the matter unification does not seem to be simply realized and the observed quark and lepton masses and mixing indeed have diverse structures. In particular, the recently revealed lepton flavor mixing is quite different from that in the quark sector [7]. To realize such diversity is therefore an interesting subject in unified theory. An available approach to this subject is to adopt flavor assignment of matter multiplets in generationdependent manner. A non-universal matter embedding naturally emerges if the unified gauge group $G_{U}$ is enough large to have the freedom to choose the ways of embedding. For example, in $E_{6}$ unified models, a vectorial representation (27-plet) doubly contains the electroweak doublet leptons (and also the down-type quarks) which cannot be distinguished with respect to the SM charges. Generally, each low-energy SM matter field $\phi$ could appear as a linear combination of some distinct fields. In this work, we consider the situation

$$
\phi=\phi^{\prime} \cos \theta_{\phi}+\phi^{\prime \prime} \sin \theta_{\phi}
$$

where the SM gauge charges of $\phi^{\prime}$ and $\phi^{\prime \prime}$ are identical to $\phi$, and these two fields are distinctive to each other with respect to $G_{U} / G_{\mathrm{SM}}$ charges. In this work, the parameter $\theta_{\phi}$ is referred to as the twisting angle for $\phi$. The flavor embedding structure is thus parametrized by twisting angles $\theta_{\phi_{i}}(i=1,2,3)$ in addition to the $G_{U} / G_{\text {SM }}$ gauge charges of $\phi^{\prime}$ and $\phi^{\prime \prime}$.

A trivial example is $G_{U}=S O(10)$ with only three 16-plets introduced as matter multiplets. That results in all twisting angles being equal. With extensions of this example, e.g. including additional matter 10-plets of $S O(10)$, the embedding of $Q_{i}, u_{i}$ and $e_{i}$ is unique for each

generation and then $\theta_{Q_{i}}=\theta_{\bar{u}_{i}}=\theta_{\bar{e}_{i}}$. On the other hand, both 16 and 10 plets contain $\overline{5}$ multiplets of $S U(5)$ subgroup, and the model contains two candidates for down-type quarks 
and doublet leptons in low-energy regime, while these candidates have different high-energy gauge charges under $U(1) \subset S O(10) / S U(5)$. In this case it is therefore natural for the twisting angles to depend on the generations; $\theta_{L_{i}} \neq \theta_{L_{j}}$ and $\theta_{\bar{d}_{i}} \neq \theta_{\bar{d}_{j}}$. $*$

In the following we discuss the explicit example of $G_{U}=E_{6}$ case [8] with three 27-plets as matter multiplets. The SM matter embedding into $E_{6}$ 27-plets and the notation are summarized in Appendix A. Our analysis is general and can be easily extended to other scenarios as long as the parametrization (2.1) is valid. The only assumption we adopt in this paper is that the gauge coupling unification with the desert is respected. That is, $S U(5)$ or larger simple group which contains $S U(5)$ as a subgroup is broken down to $G_{\mathrm{SM}}$ at the unification scale. Then the down-type quarks and doublet leptons are expressed as follows:

$$
\begin{aligned}
\bar{d}_{i} & =\overline{3}_{i}^{b} \cos \theta_{i}+\overline{3}_{i}^{c} \sin \theta_{i}, \\
L_{i} & =2_{i}^{a} \cos \theta_{i}+2_{i}^{c} \sin \theta_{i},
\end{aligned}
$$

where the common angles $\theta_{i} \equiv \theta_{\bar{d}_{i}}=\theta_{L_{i}}$ is a result of $S U(5)$ gauge symmetry. The multiplets $2^{a, c}$ and $\overline{3}^{b, c}$ are contained in a single 27 -plet and characterized by $U(1) \times U(1)^{\prime} \subset E_{6} / G_{\mathrm{SM}}$ gauge charges [e.g. see (A.5) in Appendix A]. The other assignments of SM matter fields are uniquely determined

$$
\begin{aligned}
Q_{i} & =(3,2)_{i} \\
\bar{u}_{i} & =\overline{3}_{i}^{a}, \\
\bar{e}_{i} & =1_{i}^{a} .
\end{aligned}
$$

Therefore the twisting angles for these matter fields take the universal, flavor-independent values. Finally we comment that non-parallel matter embedding discussed above is deeply suggested by the recent experimental results of neutrino flavor mixing in the context of grand unification [9] and is motivated by low-energy observations.

\subsection{Sum rules for induced scalar mass}

With the generation redundancy in unified theory discussed above, matter fields with the same SM charges generally have flavor-dependent $G_{U} / G_{\mathrm{SM}}$ quantum numbers. The corresponding flavor-violating gauge interactions leave their imprints in scalar mass spectrum in low-energy effective theory. That is determined by non-vanishing $D$-term contribution [4]

${ }^{*}$ In this work we do not consider the gauged $B-L$ symmetry which is viable only for $\theta_{i}=0$ or $\pi / 2$. The continuous values of twisting angles implies that the potential terms should possess an abelian global symmetry to avoid rapid nucleon decay. 
and renormalization-group ( $\mathrm{RG}$ ) effects [3] in high-energy theory. According to the generation structure, these effects induce specific types of non-universality for MSSM scalar mass spectrum. A notable future is that the induced non-universality is connected to the generation twisting angles discussed in the previous section, since the flavor-dependent $G_{U} / G_{\mathrm{SM}}$ charges of matter fields are parametrized by these angles. Using this fact, one can directly probe into the generation structure in high-energy theory from observed scalar mass spectrum at low-energy regime.

Here we study an example of $E_{6}$ unified theory described by (2.2) and (2.3), and examine the resultant scalar spectrum with nonzero twisting angles. The following discussion is similarly carried out for more general case with (2.1). As discussed before, the electroweak doublet leptons and down-type quarks, that is $S U(5)$ anti quintuplet matter, have a redundancy of flavor-dependent $E_{6} / G_{\mathrm{SM}}$ charges. Each twisting angle $\theta_{i}$ sets the $\overline{5}_{i}$ direction in 27 -plet and determines its $U(1)_{X} \times U(1)_{Z} \subset E_{6} / S U(5)$ quantum numbers. As a result, these scalar fields receive non-degenerate masses via the $D$-term contribution and RG effects.

Let us first consider the $D$-term contribution. It is known that the $D$-term contribution arises associated with rank reduction of gauge symmetry and its form depends on symmetry breaking pattern. There are several patterns of the unified gauge symmetry $E_{6}[10]$. However in the present case of $E_{6} \rightarrow S U(5)$, the $D$-term contribution is generally parametrized by two mass parameters without assuming explicit breaking chain (see Appendix B). For an eigenstate of $U(1)_{X} \times U(1)_{Z}$, it is given by the following form:

$$
\Delta m_{i}^{2}=\left(T_{X}\right)_{i} D_{X}+\left(T_{Z}\right)_{i} D_{Z}
$$

where $\left(T_{X}\right)_{i}$ and $\left(T_{Z}\right)_{i}$ denote the $U(1)_{X}$ and $U(1)_{Z}$ charges of the field labeled $i$, and $D_{X, Z}$ are the mass parameters which represent the deviation of the vacuum from supersymmetric flat directions. It is noted that the above parametrization is also valid in the case that $G_{U}$ is any subgroup of $E_{6}$ if one takes an appropriate limit of $D$ terms.

The equation (2.4) is readily extended to the cases where matter fields are not eigenstates of the broken $U(1)$ symmetries [i.e. $\theta_{i} \neq 0, \pi / 2$ in $\left.(2.2)\right]$. The $D$-term contributions to scalar masses of quark doublets $\left(m_{Q_{i}}^{2}\right)$, up-type quarks $\left(m_{\bar{u}_{i}}^{2}\right)$, down-type quarks $\left(m_{\bar{d}_{i}}^{2}\right)$, lepton doublets $\left(m_{L_{i}}^{2}\right)$, and charged leptons $\left(m_{\bar{e}_{i}}^{2}\right)$ are written as follows:

$$
\begin{aligned}
\Delta m_{Q_{i}}^{2}=\Delta m_{\bar{u}_{i}}^{2}=\Delta m_{\bar{e}_{i}}^{2}=-D_{X}+D_{Z} \\
\Delta m_{L_{i}}^{2}=\Delta m_{\bar{d}_{i}}^{2}=x_{i} D_{X}+z_{i} D_{Z}
\end{aligned}
$$


where the coefficients $x_{i}$ and $z_{i}$ are

$$
\begin{aligned}
x_{i} & =3 \cos ^{2} \theta_{i}-2 \sin ^{2} \theta_{i}, \\
z_{i} & =\cos ^{2} \theta_{i}-2 \sin ^{2} \theta_{i}
\end{aligned}
$$

and $D_{X}$ and $D_{Z}$ are the mass parameters. It should be noted that when one considers the cases where $S O(10)$ gauge symmetry is realized at some intermediate stage of symmetry breaking chain, then $D_{X}$ and $D_{Z}$ are directly connected to the $D$-term vacuum expectation values of $U(1)_{X}$ and $U(1)_{Z}$.

Let us first focus on the particular limit $D_{Z}=0$, which is the case that $E_{6}$ is broken down to $S O(10)$ or $S U(5) \times U(1)_{X}$ without supersymmetry-breaking corrections. With the universal hypothesis for soft terms at some high-energy scale, the induced scalar masses read from (2.5)

$$
\begin{aligned}
& m_{10}^{2} \equiv m_{Q_{i}}^{2}=m_{\bar{u}_{i}}^{2}=m_{\bar{e}_{i}}^{2}=m_{0}^{2}-D_{X} \\
& m_{\overline{5}_{i}}^{2} \equiv m_{L_{i}}^{2}=m_{\bar{d}_{i}}^{2}=m_{0}^{2}+\left(3 \cos ^{2} \theta_{i}-2 \sin ^{2} \theta_{i}\right) D_{X}
\end{aligned}
$$

at the GUT scale. From these mass expressions, we obtain a set of sum rules among threegeneration scalar masses:

$$
\begin{gathered}
\frac{m_{i j}^{2(-)}}{m_{k \ell}^{2(+)}}=\frac{\cos ^{2} \theta_{i}-\cos ^{2} \theta_{j}}{\cos ^{2} \theta_{k}+\cos ^{2} \theta_{\ell}-2 / 5} \\
m_{i j}^{2(-)}=m_{\overline{5}_{i}}^{2}-m_{\overline{5}_{j}}^{2}, \quad m_{k \ell}^{2(+)}=m_{\overline{5}_{k}}^{2}+m_{\overline{5}_{\ell}}^{2}-2 m_{10}^{2} .
\end{gathered}
$$

The similar relations follow in the $D_{X}=0$ limit;

$$
\frac{m_{i j}^{2(-)}}{m_{k \ell}^{2(+)}}=\frac{\cos ^{2} \theta_{i}-\cos ^{2} \theta_{j}}{\cos ^{2} \theta_{k}+\cos ^{2} \theta_{\ell}-2} .
$$

It is interesting to notice that these mass relations (2.9) and (2.10) are parametrized only by the twist angles and independent of the mass parameters $m_{0}^{2}$ and $D$ 's which are controlled by the detail of supersymmetry-breaking dynamics in high-energy regime. Therefore referring to these relations, one can extract clear information on the generation structure in unified theory.

As illustrative examples, consider the above sum rules for the first and second generation scalars, i.e., $(i, j)=(k, \ell)=(1,2)$. Since the numerators of the formulae are proportional to $\sin \left(\theta_{1}-\theta_{2}\right)$, the relative size of two twisting angles is important. For $\theta_{1}=0$, which corresponds 
to the case that all the first-generation matter fields originate from a single 16-plet of $S U(10)$, the scalar mass relations (2.9) and (2.10) become

$$
\frac{m_{12}^{2(-)}}{m_{12}^{2(+)}}=\left\{\begin{array}{cl}
\frac{\sin ^{2} \theta_{2}}{3 / 5+\cos ^{2} \theta_{2}} & \text { for } D_{Z}=0 \\
-1 & \text { for } D_{X}=0
\end{array}\right.
$$

On the other hand, for another limit $\theta_{1}=\pi / 2$, the relations take the forms

$$
\frac{m_{12}^{2(-)}}{m_{12}^{2(+)}}=\left\{\begin{array}{cl}
\frac{\cos ^{2} \theta_{2}}{2 / 5-\cos ^{2} \theta_{2}} & \text { for } D_{Z}=0 \\
\frac{\cos ^{2} \theta_{2}}{1+\sin ^{2} \theta_{2}} & \text { for } D_{X}=0
\end{array}\right.
$$

For the latter case $\left(D_{X}=0\right)$ in (2.11), the $\theta_{2}$ dependence drops out from the sum rule. This is because, due to the absence of $S O(10)$-violating parameters $\theta_{1}$ and $D_{X}$, the gauge symmetry ensures the degeneracy of the first-generation scalar masses $m_{\overline{5}_{1}}^{2}$ and $m_{10_{1}}^{2}$. Also a similar observation appears in the case $D_{Z}=0$, where the $\theta_{2}$ dependence vanishes for $\cos ^{2} \theta_{1}=1 / 5$.

There generally appear the corrections to scalar soft masses from RG evolution above the unification scale. For the contributions from GUT gauge interactions, scalar masses generally receive the gaugino corrections according to their $E_{6} / G_{\mathrm{SM}}$ charges. Therefore the non-degeneracy among different $\overline{5}_{i}$ matter scalars which survive to low-energy regime depends only on the differences among the twisting angles:

$$
\begin{aligned}
m_{\overline{5}_{i}}^{2} & =m_{\overline{5}(16)}^{2} \cos ^{2} \theta_{i}+m_{\overline{5}(10)}^{2} \sin ^{2} \theta_{i} \\
& =m_{0}^{2}+\tilde{D} \cos ^{2} \theta_{i}
\end{aligned}
$$

where $m_{\overline{5}(16)}^{2}\left[m_{\overline{5}(10)}^{2}\right]$ is the supersymmetry-breaking mass parameter for $\overline{5}$ scalar fields which belong to 16-plets (10-plets) of $S O(10)$. It should be noted that, even when the flavorviolating effects from $D$-term contribution and $R G$ evolution are included, we keep the form (2.13) without the loss of generality, taking a suitable redefinition of $m_{0}^{2}$ and $\tilde{D}$. As a result, a set of general sum rules among the masses of $\overline{5}$ scalar fields are obtained:

$$
\begin{gathered}
\frac{m_{i j}^{2(-)}}{m_{k \ell n}^{2}}=\frac{\cos ^{2} \theta_{i}-\cos ^{2} \theta_{j}}{\cos ^{2} \theta_{k}+\cos ^{2} \theta_{\ell}-2 \cos ^{2} \theta_{n}}, \\
m_{k \ell n}^{2}=m_{\overline{5}_{k}}^{2}+m_{\overline{5}_{\ell}}^{2}-2 m_{\overline{5}_{n}}^{2} .
\end{gathered}
$$

Note that the relation becomes trivial if some of the generation indices take a common value in the denominator (or in the numerator). That is, (2.14) is meaningful only when $k \neq \ell \neq n \neq k$, 
and therefore necessarily includes the third generation matter. Like the relations (2.9) and (2.10), this mass relation is also independent of the high-energy free parameters $m_{0}$ and $\tilde{D}$, and therefore useful to directly probe the flavor structure in high-energy theory.

In the present example, the quark doublets, up-type quarks and charged leptons have no twists and the induced spectrum is universal at the boundary scale. If future particle experiments would observe some deviations from such universality, that would provide an evidence that the unified theory has non-trivial and more complicated matter structure beyond the simplest one discussed in this paper.

\section{$3 \quad$ Probing flavor structure with low-energy observables}

In this section, we perform the extraction of flavor structure in unified theory through the twisting angles using the sum rules (2.9), (2.10) and (2.14) among sfermion soft masses. The scalar mass corrections are induced by the gauge interactions of $G_{U} / G_{\mathrm{SM}}$ symmetry and have imprints of high-energy flavor theory. The sum rules are useful in that they are independent of the overall mass scale and the sizes of $D$-term corrections. Therefore the generation structure is directly probed from scalar mass parameters which would be expected to be in principle revealed in future particle experiments.

We start from the discussion about RG effects on the first and second generation scalar masses between the unification scale $M_{G}$ and the electroweak scale $M_{S}$. For the first two generations, the unification-scale soft masses at one-loop order are written in terms of lowenergy scalar masses and the universal gaugino mass parameter $M_{1 / 2}$ as follows:

$$
\begin{aligned}
m_{i}^{2}\left(M_{G}\right) & =m_{i}^{2}\left(M_{S}\right)+\xi_{i} M_{1 / 2}^{2}+\eta_{i} S\left(M_{S}\right), \\
\xi_{i} & =\sum_{n} \frac{2}{b_{n}} C_{2}^{n}(i)\left(\frac{g_{n}^{4}\left(M_{S}\right)}{g_{G}^{4}}-1\right), \\
\eta_{i} & =\frac{3}{5 b_{1}}\left(T_{Y}\right)_{i}\left(\frac{g_{G}^{2}}{g_{1}^{2}\left(M_{S}\right)}-1\right) .
\end{aligned}
$$

Here the indices $n=1,2,3$ denote the SM gauge groups with the coupling constants $g_{n}$, which meet to the universal value $g_{G}$ at $M_{G}$. The gauge beta function coefficients are given by $b_{n}=(-3,1,33 / 5)$ for the MSSM, and $C_{2}^{n}(i)$ is the quadratic Casimir invariant for the scalar labeled $i$. The mass parameter $S$ is the hypercharge $D$-term given by $S=\operatorname{Tr}\left[\left(T_{Y}\right)_{i} m_{i}^{2}\right]$.

It is noted that the high-scale soft masses (3.1) are described only by the observables at future experiments; the gauge couplings, gaugino masses, and sfermion masses. For example, 
the universal gaugino mass is obtained by converting the 'observed' gluino mass through the RG equations as $M_{1 / 2} \simeq M_{3} / 2.5$ (or similarly from the neutralino or chargino masses). The Higgs soft masses are relevant to the electroweak symmetry breaking, which we do not focus on in this paper. The low-energy value of $S$ could be extrapolated by the observed up-quark and electron scalar masses in this case and we simply set $S=0$ in the following analysis. Using the RG solution (3.1), the mass combinations which appear in the sum rules (2.9) and (2.10) are written by

$$
\begin{aligned}
m_{12}^{2(-)} & =m_{L_{1}}^{2}\left(M_{S}\right)-m_{L_{2}}^{2}\left(M_{S}\right) \\
& =m_{\bar{d}_{1}}^{2}\left(M_{S}\right)-m_{\bar{d}_{2}}^{2}\left(M_{S}\right), \\
m_{12}^{2(+)} & =m_{L_{1}}^{2}\left(M_{S}\right)+m_{L_{2}}^{2}\left(M_{S}\right)-2 m_{\bar{e}_{1}}^{2}\left(M_{S}\right)-2\left(\xi_{L}-\xi_{e}\right) M_{1 / 2}^{2} \\
& =m_{\bar{d}_{1}}^{2}\left(M_{S}\right)+m_{\bar{d}_{2}}^{2}\left(M_{S}\right)-2 m_{\bar{e}_{1}}^{2}\left(M_{S}\right)-2\left(\xi_{d}-\xi_{e}\right) M_{1 / 2}^{2} .
\end{aligned}
$$

The coefficients of $\mathrm{RG}$ gauge contribution are evaluated by the one-loop $\mathrm{RG}$ equations for gauge couplings; $\xi_{L} \simeq 0.49, \xi_{e} \simeq 0.15$ and $\xi_{d} \simeq 4.35$ for $M_{S} \simeq 1 \mathrm{TeV}$.

The twist angles for the first and second generations now become the functions of mass parameters of scalar leptons (and down-type quarks). In Fig. 1, we show using the formulae (2.9) and (2.10) the extracted twisting angles from the low-energy non-degeneracy of scalar leptons. For degenerate scalar leptons, the twist angles are identical $\left(\theta_{1}=\theta_{2}\right)$. In general, a larger mass difference implies a larger deviation from the naive alignment $\theta_{1}=\theta_{2}$. Notice however that there exist the points where the two angles $\theta_{1}$ and $\theta_{2}$ are independent to each other. For the case with (2.9) $[(2.10)]$, that is given by $\theta_{1}=\arccos \left(\frac{1}{\sqrt{5}}\right)\left[\theta_{1}=0\right]$, at which scalar masses of lepton doublets and charged leptons in the first generation are common at the unification scale. Such a behavior is not affected by the overall scale of superparticle masses. A mass-dependent irregular line also appears if $\sqrt{m_{L_{1}}^{2}}-\sqrt{m_{L_{2}}^{2}} \simeq 120 \mathrm{GeV}$, where $\theta_{2}=\pi / 2$ is predicted for any value of $\theta_{1}$. This is the point that the masses of the second-generation scalar leptons are unified.

Next let us examine the formula (2.14). The third generation scalars are necessarily incorporated in the analysis. They generally receive non-negligible Yukawa effects through the RG evolution. The size of RG Yukawa effects depend on $\tan \beta$ which is the ratio of upand down-type Higgs vacuum expectation values. For a sufficiently small value of $\tan \beta$, the bottom and tau Yukawa couplings become small, $y_{b, \tau} \ll 1$, and the RG contribution to scalar masses is negligible. In this case, all the RG effects shown in (3.1) are canceled out in the mass formula (2.14). In particular, the gaugino masses are completely irrelevant to the analysis. 


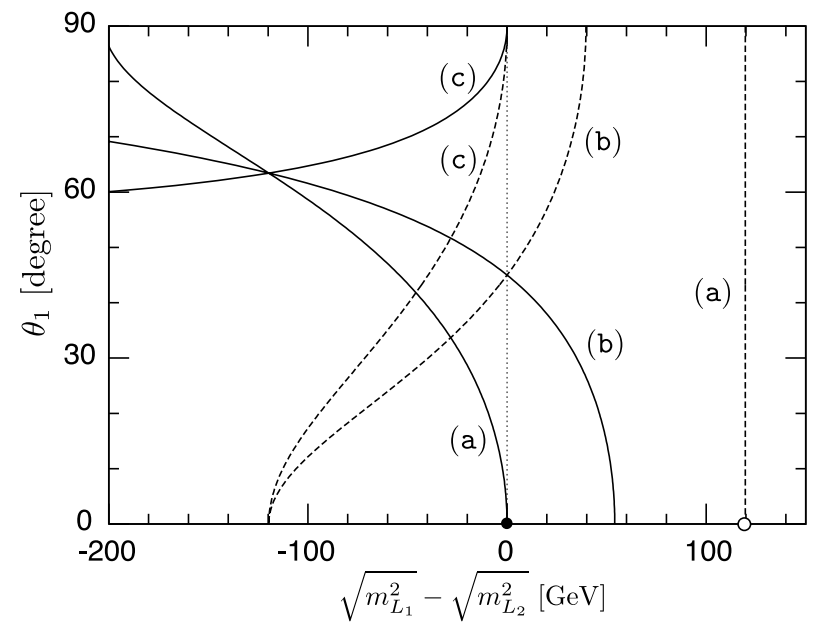

Figure 1: The extracted values of twisting angles from the first and second generation scalar lepton doublets. The solid (dashed) lines are obtained from the formulae (2.9) $[(2.10)]$, and the lines (a), (b), (c) correspond to $\theta_{2}=0, \pi / 4, \pi / 2$, respectively. The gaugino and scalar masses are fixed as $M_{1 / 2}=300 \mathrm{GeV}, m_{L_{1}}^{2}\left(M_{S}\right)+m_{L_{2}}^{2}\left(M_{S}\right)=2 \times(500 \mathrm{GeV})^{2}$, and $m_{e_{1}}^{2}\left(M_{S}\right)=$ $(400 \mathrm{GeV})^{2}$.

We can extract the twisting angles from low-energy masses of scalar leptons as

$$
\frac{\cos ^{2} \theta_{1}-\cos ^{2} \theta_{2}}{\cos ^{2} \theta_{1}+\cos ^{2} \theta_{2}-2 \cos ^{2} \theta_{3}}=\frac{m_{L_{1}}^{2}\left(M_{S}\right)-m_{L_{2}}^{2}\left(M_{S}\right)}{m_{L_{1}}^{2}\left(M_{S}\right)+m_{L_{2}}^{2}\left(M_{S}\right)-2 m_{L_{3}}^{2}\left(M_{S}\right)} .
$$

A similar result follows for scalar down-type quarks. Fig. 2 shows the correlation between the twisting angles of the first and second generation scalar leptons. It is found from the figure that the non-degenerate mass spectrum implies a deviation from the naive alignment of twisting angles. The results are also sensitive to the third-generation matter structure. As typical examples, we show the cases that $\theta_{3}=0$ and $\pi / 2$.

If $\tan \beta$ is not so small enough to neglect the Yukawa dependent RG effects to the third generation scalar masses, one should evaluate the contribution to the linear combination $m_{123}^{2}$. That is carried out by solving the MSSM RG equations numerically. In Fig. 3, we show the prediction of twisting angles for $\tan \beta=10$. The scalar masses (difference) and the generation angles are found to be tightly correlated even when the Yukawa RG effects are taken into account.

In the present example, the relative size of non-degeneracy between scalar leptons and down-type quarks is connected by the $S U(5)$ symmetry. Since the twist redundancy appears only for anti quintuplet matter, the other MSSM matter scalars in the first and second 

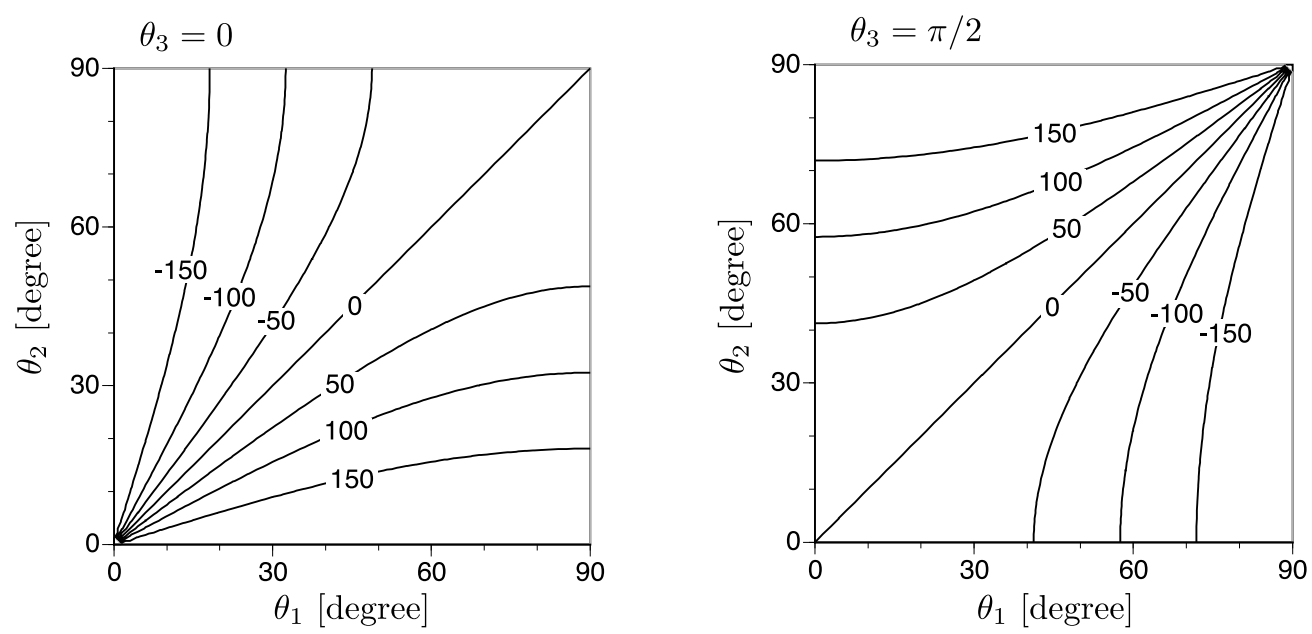

Figure 2: The correlation between the twist angles $\theta_{1}$ and $\theta_{2}$ indicated by the sum rule (3.4). The mass difference $\sqrt{m_{L_{1}}^{2}}-\sqrt{m_{L_{2}}^{2}}$ is shown in the figures. The scalar masses are fixed as $m_{L_{1}}^{2}\left(M_{S}\right)+m_{L_{2}}^{2}\left(M_{S}\right)=2 \times(500 \mathrm{GeV})^{2}$ and $m_{L_{3}}^{2}\left(M_{S}\right)=(400 \mathrm{GeV})^{2}$ as an example.

generations are expected to obtain the degenerate masses. Such a significant pattern of nonuniversality is a particular prediction and interesting signal of the $E_{6}$ unified theory with twisted matter structure in a 27-plet.

If there were other sizable flavor-violating contributions to scalar masses in unified theory (e.g. flavor-dependent RG effects), then the mass relations are disturbed. Such contributions may be extracted under model-dependent assumptions and is expected to be small, in particular, for the first and second generations. Another possible source of violation is a gauged flavor symmetry, often introduced in order to explain the observed Yukawa coupling hierarchy. The contributions from flavor gauge sector ( $D$ term and gaugino mass) [11] can also be treated in a similar manner to the above analysis as long as the parametrization (2.1) holds. Further the $D$-term corrections which arise in the electroweak symmetry breaking are not included in the analysis. This is due to the fact that such effects are always canceled out in the combinations $m_{i j}^{2(-)}$ and $m_{k \ell n}^{2}$, and for $m_{12}^{2(+)}$ the contribution is generally small. These factors can be straightforwardly incorporated into the analysis, if desired, at the cost of complexity.

In this section, we demonstrate that the generation structure in unified theory is directly examined by using the information of sfermion mass parameters which would be observed in future experiments. The analysis can be generalized to other unified model examples. The 


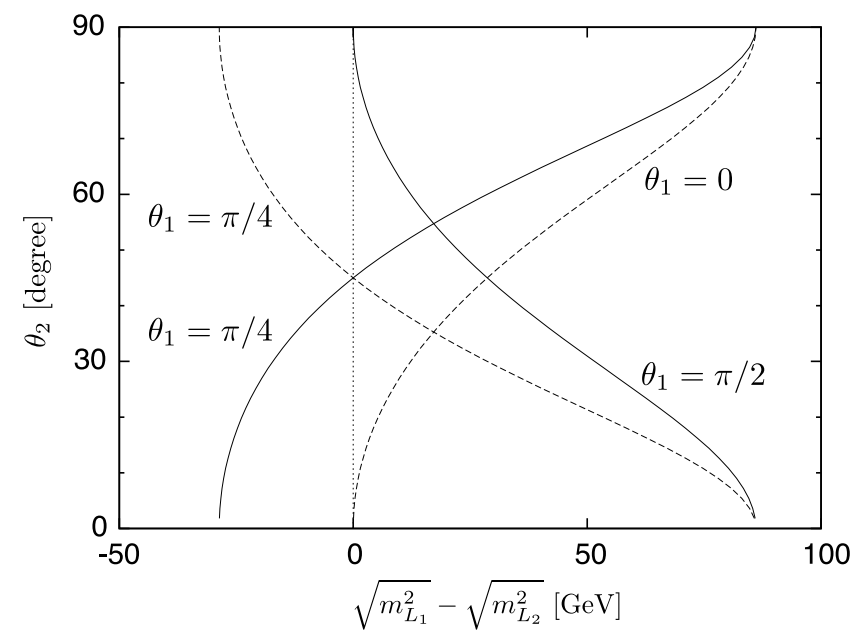

Figure 3: A twisting angle as the function of the mass difference between the first two generations scalar leptons. The solid (dashed) lines represent the case that $\theta_{3}=0\left(\theta_{3}=\pi / 2\right)$. We assume $\tan \beta=10$ and $M_{1 / 2}=300 \mathrm{GeV}$. The low-energy scalar lepton masses are fixed as $m_{L_{1}}^{2}\left(M_{S}\right)+m_{L_{2}}^{2}\left(M_{S}\right)=2 \times(700 \mathrm{GeV})^{2}, m_{e_{1}}^{2}\left(M_{S}\right)=(500 \mathrm{GeV})^{2}$, and $m_{L_{3}}^{2}\left(M_{S}\right)=(650 \mathrm{GeV})^{2}$.

scalar mass spectrum in low-energy regime and their various sum rules offer useful insights into un-revealed flavor dynamics in high-energy fundamental theory.

\section{Flavor twists and flavor violation}

In widely studied MSSM parameter space, for instance, with the universal mass parameters at the unification scale, the scalar masses of the first two generations are degenerate. Such a degeneracy is the most naive and often adopted hypothesis for evading the experimental constraints from flavor-violating processes [12] so that non-SM sources of flavor violation do not exist at the unification scale. Namely, off-diagonal elements of sfermion mass matrices vanish in the super-CKM (SCKM) basis, which diagonalizes the (scalar)-(fermion)-(gaugino) vertex for mass eigenstates of matter fermions. In this case, the flavor violation triggered by superparticles only appears via quantum corrections, which are often small enough to avoid the experimental bounds.

With the generation redundancy of matter embedding (2.1), scalar fields generally realize non-degenerate mass spectrum. Such a non-degeneracy does not ensure vanishing off-diagonal elements of scalar mass matrices in the SCKM basis. In this section, we study the experimental limitation on matter flavor structure in unified theory, e.g. the twisting angles in the 
$E_{6}$ example (2.2). The constraints come from flavor-violating rare processes, in particular, focusing on the charged lepton sector. A similar analysis can also be performed with the general case (2.1).

To study the constraints on generation structure, one should fix the relation between the two flavor bases: in one basis the Yukawa interactions are diagonalized and in the other basis scalar soft mass matrices are. We call the basis where scalar mass matrices have no off-diagonal entries as "the scalar basis". In the following, we treat the mass matrix for scalar lepton doublets, which matrix is relevant for the flavor-violating rare processes in the lepton sector. To turn to the SCKM basis from the scalar one, the scalar lepton mass matrix is rotated as

$$
m_{L_{i j}}^{2}=\left(\begin{array}{ccc}
m_{L_{1}}^{2} & & \\
& m_{L_{2}}^{2} & \\
& & m_{L_{3}}^{2}
\end{array}\right) \rightarrow m_{L_{i j}}^{2(\mathrm{SCKM})}=V_{e L}\left(\begin{array}{ccc}
m_{L_{1}}^{2} & & \\
& m_{L_{2}}^{2} & \\
& & m_{L_{3}}^{2}
\end{array}\right) V_{e L}^{\dagger}
$$

where $V_{e L}$ is defined by the charged-lepton Yukawa matrix $\left(Y_{e}\right)_{i j}$ in the scalar basis as

$$
Y_{e} Y_{e}^{\dagger}=V_{e L}^{\dagger}\left(\begin{array}{lll}
\left|y_{e}\right|^{2} & & \\
& \left|y_{\mu}\right|^{2} & \\
& & \left|y_{\tau}\right|^{2}
\end{array}\right) V_{e L}
$$

The lepton mixing matrix [13] is defined by

$$
V_{\mathrm{MNS}}=V_{e L} V_{\nu}^{\dagger}
$$

where $V_{\nu}$ rotates the left-handed neutrinos such that the neutrino mass matrix is diagonalized.

The recent neutrino experiments have revealed that the lepton mixing matrix $V_{\mathrm{MNS}}$ has relatively large 1-2 and 2-3 mixing, and on the other hand, the 1-3 mixing angle is found to be small [7]. These experimental results on the lepton mixing matrix, however, does not directly restrict $V_{e L}$ alone as long as the form of $V_{\nu}$ is undetermined. It is natural to imagine that the observed large mixing angles have definite origins. In this case, there are three possible patterns: (i) $V_{e}\left(V_{\nu}\right)$ has large 2-3 (1-2) angle, (ii) $V_{e}$ has both large mixing angles, and (iii) $V_{\nu}$ has both large mixing angles. The last pattern may be interesting in that the tiny neutrino mass generation simultaneously enhances the neutrino mixing angles [14] while charged field mixings remain small. In this section, however, we do not consider the case (iii) since no significant requirement on twisting angles cannot be obtained. Thus the experimental bounds on non-aligned twisting angles depend on the form of charged lepton mixing $V_{e L}$.

Let us examine the first case that $V_{e}$ has large 2-3 mixing angle (the large 1-2 mixing 
comes from the neutrino sector). We explicitly consider the following form of $V_{e L}$ :

$$
V_{e L}=\left(\begin{array}{ccc}
1 & \epsilon_{x} & \epsilon_{z} \\
\frac{-\epsilon_{x}+\epsilon_{z}}{\sqrt{2}} & \frac{1}{\sqrt{2}} & \frac{-1}{\sqrt{2}} \\
\frac{-\epsilon_{x}-\epsilon_{z}}{\sqrt{2}} & \frac{1}{\sqrt{2}} & \frac{1}{\sqrt{2}}
\end{array}\right)
$$

where the 2-3 generation mixing is maximal and the other ones $\epsilon_{x}$ and $\epsilon_{z}$ are assumed to be small. The situation is compatible with tiny 1-3 mixing without artificial fine tuning in $V_{\text {MNS }}$. With this form of $V_{e L}$, the scalar lepton mass matrix in the SCKM basis is given by

$$
m_{L}^{2(\mathrm{SCKM})} \simeq\left(\begin{array}{ccc}
m_{L_{1}}^{2} & \frac{\epsilon_{x} m_{21}^{2(-)}-\epsilon_{z} m_{31}^{2(-)}}{\sqrt{2}} & \frac{\epsilon_{x} m_{21}^{2(-)}+\epsilon_{z} m_{31}^{2(-)}}{\sqrt{2}} \\
\frac{\epsilon_{x} m_{21}^{2(-)}-\epsilon_{z} m_{31}^{2(-)}}{\sqrt{2}} & \frac{m_{21}^{2(-)}+m_{31}^{2(-)}}{2} & \frac{m_{23}^{2(-)}}{2} \\
\frac{\epsilon_{x} m_{21}^{2(-)}+\epsilon_{z} m_{31}^{2(-)}}{\sqrt{2}} & \frac{m_{23}^{2(-)}}{2} & \frac{m_{21}^{2(-)}+m_{31}^{2(-)}}{2}
\end{array}\right) .
$$

The off-diagonal elements of the mass matrix are relevant for lepton flavor violating processes such as $\ell_{j} \rightarrow \ell_{i} \gamma \doteq$ Among them, $\mu \rightarrow e \gamma$ tends to give the most severe constraints on the flavor non-degeneracy, for which process the relevant off-diagonal matrix element is

$$
m_{L_{12}}^{2(\mathrm{SCKM})}=\frac{\epsilon_{x} m_{21}^{2(-)}-\epsilon_{z} m_{31}^{2(-)}}{\sqrt{2}} .
$$

A larger value of off-diagonal matrix elements increases the decay amplitude. In other words, if $\epsilon_{x, z}$ are not suppressed, the flavor non-universality $m_{12}^{2(-)}$ and $m_{13}^{2(-)}$, i.e. the twisting angles are strongly constrained unless a cancellation occurs between the two terms in the righthanded side of (4.6).

In Fig. 4, we show typical correlations between the $\ell_{j} \rightarrow \ell_{i} \gamma$ branching fractions and twisting angles. The present experimental upper bounds on the fractions are $\operatorname{BR}(\mu \rightarrow e \gamma)<$ $1.2 \times 10^{-11}, \operatorname{BR}(\tau \rightarrow \mu \gamma)<6.8 \times 10^{-8}$ and $\operatorname{BR}(\tau \rightarrow e \gamma)<1.1 \times 10^{-7}$ at the $90 \%$ confidence level [16]. The validity of scenarios depends on the mixing parameters $\epsilon_{x}$ and $\epsilon_{z}$. We here examine two typical examples: the solid and dashed lines in the figure represent the cases that $\left(\epsilon_{x}, \epsilon_{z}\right)=\left(10^{-2.3}, 10^{-2.5}\right)$ and $\left(0,10^{-2}\right)$, respectively. At first, generally speaking, the $\mu \rightarrow e \gamma$ process gives the most stringent constraint on the twisting angles. For the solid-line case, there exists a parameter region where the cancellation between the two terms in (4.6) occurs. That decreases the $\mu \rightarrow e \gamma$ branching ratio and a large generation twisting is generally allowed. For the dashed-line case, the prediction is slightly larger than the experimental limit and

\footnotetext{
${ }^{\dagger}$ If neutrinos have sizable Yukawa couplings, the off-diagonal elements in $m_{L}^{2}$ are radiatively generated via RG evolution down to the decoupling scale of right-handed neutrinos, and the induced lepton flavor violation has been investigated in the literature [15]. For simplicity we do not consider this issue in the present analysis.
} 


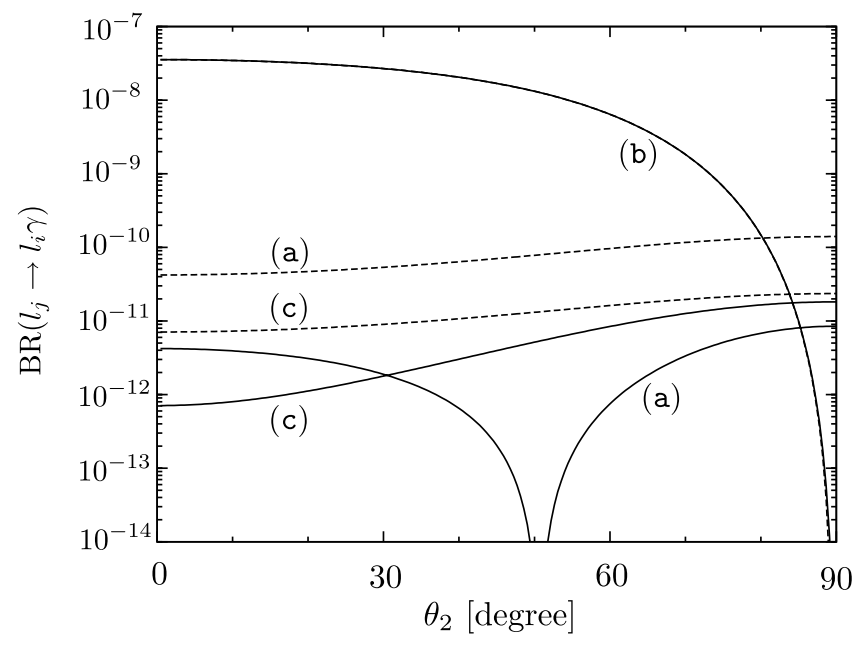

Figure 4: Predictions of flavor-violating rare decay of charged leptons: $\mu \rightarrow e \gamma$ [Line (a)], $\tau \rightarrow \mu \gamma$ [Line (b)] and $\tau \rightarrow e \gamma\left[\right.$ Line (c)]. The solid lines show the case with $\left(\epsilon_{x}, \epsilon_{z}\right)=$ $\left(10^{-2.3}, 10^{-2.5}\right)$ and the dashed ones with $\left(\epsilon_{x}, \epsilon_{z}\right)=\left(0,10^{-2}\right)$. The $\tau \rightarrow \mu \gamma$ branching fraction is same for both cases. The twist angles are set as $\theta_{1}=0$ and $\theta_{3}=\pi / 2$ as examples and superparticle masses are taken as in Fig. 3.

the scenario is marginally excluded. However in case that $\epsilon_{x}$ is sufficiently small, the $\mu \rightarrow e \gamma$ amplitude rather depends on $\epsilon_{z}$ and is suppressed as $\operatorname{BR}(\mu \rightarrow e \gamma) \lesssim 10^{-12}$ for $\epsilon_{z} \lesssim 10^{-3}$. That does not lead to any experimental constraints. Secondly, the $\tau \rightarrow \mu \gamma$ process is relevant to the non-degeneracy between the second and third generations. Its amplitude is enhanced for $\theta_{2} \rightarrow 0$ where the predicted branching ratio is just below the present experimental bound and would be testable in near future experiments. The $\tau \rightarrow \mu \gamma$ decay is now induced through the large 2-3 generation mixing in $V_{e L}$ and therefore the constraint on matter twist is insensitive to the mixing parameters $\epsilon_{x}$ and $\epsilon_{z}$. Finally the $\tau \rightarrow e \gamma$ process strongly depends on the details of $\epsilon_{x}$ and $\epsilon_{z}$. But we found that the branching fraction is predicted to be much smaller than the experimental bound and does not give any meaningful constraints on the generation twisting as long as $\epsilon_{z}$ is small, as observed in the neutrino experiments.

The second case we discuss is that $V_{e L}$ is the dominant origin for both large lepton mixing angles. However, it is found in this case that the scalar lepton non-degeneracy of the first two generations is severely constrained by the experimental limit from the $\mu \rightarrow e \gamma$ rare process. As a result, the twisting angles $\theta_{1}$ and $\theta_{2}$ should be almost aligned even if the other mixing angles in $V_{e L}$ are sufficiently small. To see this behavior, we show in Fig. 5 the $\mu \rightarrow e \gamma$ branching ratio as a function of the 1-2 lepton mixing and twisting angles. In the figure we 


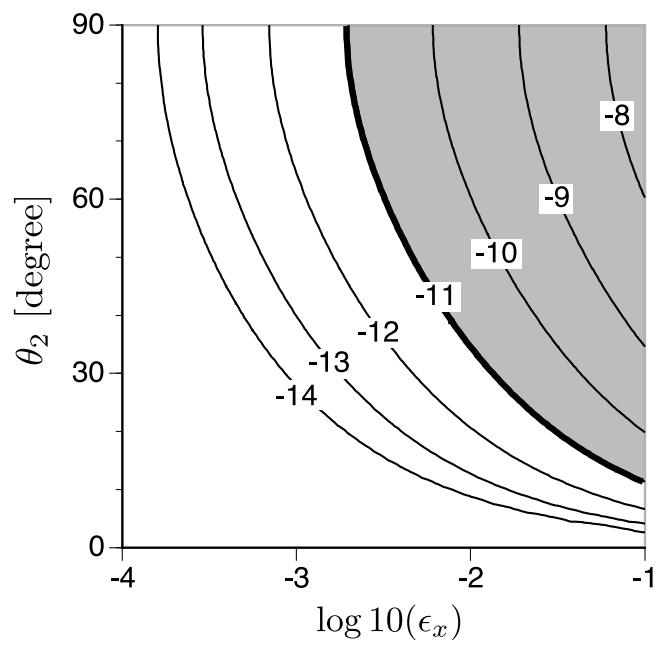

Figure 5: Typical predictions for the $\mu \rightarrow e \gamma$ branching ratio. The twisting angles are set to $\theta_{1}=0$ and $\theta_{3}=\pi / 2$, and $V_{e L}$ has the form (4.7) with $\epsilon_{y}=\epsilon_{z}=10^{-2}$. The model parameters are similarly taken as in Fig. 4, The gray shaded area is excluded by the current experimental upper bound.

take the explicit form of $V_{e L}$ as

$$
V_{e L} \simeq\left(\begin{array}{ccc}
1 & \epsilon_{x} & \epsilon_{z} \\
-\epsilon_{x} & 1 & \epsilon_{y} \\
-\epsilon_{z} & -\epsilon_{y} & 1
\end{array}\right)
$$

with $\epsilon_{y}=\epsilon_{z}=10^{-2}$. That is, we have assumed a conservative limit that the irrelevant lepton mixing angles are tiny $\left(\epsilon_{y, z} \ll 1\right)$, and then examine the $\epsilon_{x}$ dependence. From the figure, one easily sees that the constraint from the $\mu \rightarrow e \gamma$ decay is rather severe for $\epsilon_{x}$, even when the other mixing angles in $V_{e L}$ are small. Though the other processes, $\tau \rightarrow \mu \gamma$ and $\tau \rightarrow e \gamma$, are sensitive to $\epsilon_{y}$ and $\epsilon_{z}$, the amplitudes are not more than those in Fig. 4 ,

The above discussion depends on supersymmetry-breaking soft mass parameters. It is well known that the decay rates are enhanced for a large value of $\tan \beta$ [15]. Therefore for larger $\tan \beta$, the constraints on the generation twisting become severer than the cases in Figs. 4 and 5. The decay rates also have dependences on superparticle mass scale; lighter superparticles enhance the amplitudes.

In this section, we have examined the flavor-violating rare decays of charged leptons in the presence of non-trivial generation structure in high-energy unified theory. The results depend on the form of charged lepton Yukawa matrix in the flavor basis where scalar lepton mass matrices are diagonalized. If one generally considers other forms of non-degeneracy (2.1) and 
other flavor violation processes, the analysis can be performed in completely parallel fashion.

\section{Summary}

In this paper, we have demonstrated that the flavor structure in unified theory is probed with superparticle mass spectrum in low-energy regime. The flavor dependence is parametrized by the introduction of twisting angles which represent the embedding redundancy of SM matter multiplets into unified theory. The non-universality is generally induced in supersymmetrybreaking sfermion soft masses by grand unified gauge interactions. We have obtained several sum rules for induced scalar masses: (2.9), (2.10) and (2.14). These relations are independent of explicit mass scales and are the functions of twist angles only. Therefore the relations are useful to directly probe the flavor sector in high-energy regime.

Using the mass relations, we have studied the twist angle behaviors in the explicit $E_{6}$ example. It has been found that matter flavor structure in unified theory is explored if future particle experiments would reveal the superparticle mass parameters at low energy. The physical implications from flavor-violating decay processes have also been analyzed. The results depend on the form of Yukawa matrices in the flavor basis where scalar lepton mass matrices are diagonalized. It has been shown that non-aligned twist angles are compatible with the experimental limits from lepton flavor violation as long as there is no large offdiagonal elements of charged-lepton Yukawa matrix between the first two generations.

The embedding redundancy naturally emerges if the fundamental unified gauge theory is $S O(10)$ or larger, and/or with the presence of extra matter. Future feedback from future particle experiments would reveal specific patterns of sfermion mass spectrum, with which we would have novel insights into the flavor structure in fundamental theory.

\section{Acknowledgments}

This work is supported by grant-in-aid for the scientific research on the priority area (\#441) "Progress in elementary particle physics of the 21st century through discoveries of Higgs boson and supersymmetry" (No. 16081209) and by a scientific grant from Monbusho (No. 17740150).

\footnotetext{
${ }^{\ddagger}$ For supersymmetry breaking parameters, a known scenario which could directly probe high-energy property of the model is that the RG modification and supersymmetric threshold corrections are complemented by the contribution from conformal anomaly [17].
} 


\section{Appendix}

\section{A SM matter in $E_{6}$ multiplet}

In this appendix, we summarize the SM matter assignment in unified theory, in particular, the embedding into a 27-plet of $E_{6}$ gauge group. A main subject here is to show that the embedding of right-handed down-type quarks and electroweak doublet leptons (and also righthanded neutrinos) is not uniquely determined and can be generation dependent, which fact leads to non-universal quantum numbers of the broken gauge symmetry $E_{6} / G_{\mathrm{SM}}$.

In order to examine possible matter assignments, it is useful to consider the maximal subgroup decomposition of the vector representation 27 under $E_{6} \supset S U(3)_{C} \times S U(3)_{L} \times$ $S U(3)_{R}:$

$$
27=(3,3,1) \oplus(\overline{3}, 1, \overline{3}) \oplus(1, \overline{3}, 3) .
$$

The first factor $S U(3)_{C}$ is identified with the $S U(3)$ color group of the SM. Furthermore, under the fixing of electroweak $S U(2)$ symmetry s.t. $S U(3)_{L} \supset S U(2)_{L}$, each representation of $\left(S U(3)_{C}, S U(3)_{L}, S U(3)_{R}\right)$ is decomposed as

$$
\begin{aligned}
(3,3,1) & =(3,2,1) \oplus(3,1,1) \equiv(3,2) \oplus 3 \\
(\overline{3}, 1, \overline{3}) & =(\overline{3}, 1, \overline{3}) \equiv \overline{3}^{\alpha} \\
(1, \overline{3}, 3) & =(1,2,3) \oplus(1,1,3) \equiv 2^{\alpha} \oplus 1^{\alpha} .
\end{aligned}
$$

Here we have used the notation that the labels of trivial representation are dropped, and the superscript $\alpha$ represents the $S U(3)_{R}$ index. Thus the components in a 27-plet transform in the following way under $E_{6} \supset S U(3)_{C} \times S U(2)_{L}$;

$$
27=(3,2) \oplus 3 \oplus \overline{3}^{\alpha} \oplus 2^{\alpha} \oplus 1^{\alpha}
$$

where $i=1,2,3$ denote the generation indices.

It is found that the $(3,2)$ multiplet is the unique choice for electroweak doublet quarks in the SM. On the other hand, each 27-plet contains three sets of $\overline{3}, 2$ and 1 , which correspond to $S U(3)_{R}$ triplets, and therefore the embedding of other SM matter fields is not uniquely identified. The components of $S U(3)_{R}$ triplets are discriminated with respect to the quantum numbers of three $U(1)$ symmetries $[U(1)]^{3} \subset E_{6} / S U(3)_{C} \times S U(2)_{L}$. We take a basis for these 
three $U(1)$ symmetries as

$$
\begin{aligned}
E_{6} & \supset S O(10) \times U(1)_{Z} \\
& \supset S U(5) \times U(1)_{X} \times U(1)_{Z} \\
& \supset S U(3)_{C} \times S U(2)_{L} \times U(1)_{V} \times U(1)_{X} \times U(1)_{Z} .
\end{aligned}
$$

In a certain charge normalization, the $S U(3)_{R}$ singlets $(3,2)$ and 3 are found to have the following quantum numbers of $\left(U(1)_{V}, U(1)_{X}, U(1)_{Z}\right)$ symmetry:

$$
Q((3,2))=(1 / 6,-1,1), \quad Q(3)=(-1 / 3,2,-2)
$$

With this normalization, the $S U(3)_{R}$ triplets $\overline{3}^{\alpha}, 2^{\alpha}$ and $1^{\alpha}$ take the following $U(1)$ charge assignment:

$$
\begin{array}{llll}
Q\left(\overline{3}^{a}\right)=(-2 / 3,-1,1), & Q\left(2^{a}\right)=(-1 / 2,3,1), & & Q\left(1^{a}\right)=(1,-1,1), \\
Q\left(\overline{3}^{b}\right)=(1 / 3,3,1), & Q\left(2^{b}\right)=(1 / 2,2,-2), & & Q\left(1^{b}\right)=(0,-5,1), \\
Q\left(\overline{3}^{c}\right)=(1 / 3,-2,-2), & Q\left(2^{c}\right)=(-1 / 2,-2,-2), & Q\left(1^{c}\right)=(0,0,4) .
\end{array}
$$

(For the proper normalization of $E_{6}$ generators, these three $U(1)$ charges are multiplied by $\sqrt{3 / 5}, \sqrt{1 / 40}, \sqrt{1 / 24}$, respectively). It is easily found from this assignment that the $S U(3)_{R}$ triplets $\overline{3}^{\alpha}, 2^{\alpha}$ and $1^{\alpha}$ belong to the multiplets of $S O(10)$ as

$$
27=16 \oplus 10 \oplus 1, \quad \begin{aligned}
16 & \ni \overline{3}^{a}, \overline{3}^{b}, 2^{a}, 1^{a}, 1^{b}, \\
10 & \ni \overline{3}^{c}, 2^{b}, 2^{c}, \\
1 & \ni 1^{c} .
\end{aligned}
$$

The last factor undetermined is the hypercharge symmetry $U(1)_{Y}$ in the SM. In general, the generator of $U(1)_{Y}$ is a linear combination of the above three $U(1)$ generators. The successful gauge coupling unification in the MSSM implies that the SM gauge group $G_{\mathrm{SM}}=$ $S U(3)_{C} \times S U(2)_{L} \times U(1)_{Y}$ is embedded into a simple group at the unification scale. Under this situation, it is a natural assumption that $E_{6}$ is broken down to a subgroup which contains $S U(5)$ symmetry at a high-energy scale, and consequently the hypercharge $U(1)_{Y}$ is identified with $U(1)_{V}$ in the above decomposition of $E_{6}$ symmetry.

As a result, the residual SM matter fields, up-type quarks $\bar{u}_{i}$, down-type quarks $\bar{d}_{i}$, doublet 
leptons $L_{i}$ and charged leptons $\bar{e}_{i}$, are found to be uniquely embedded into 27-plets as follows:

$$
\begin{aligned}
\bar{u}_{i} & =\overline{3}_{i}^{a}, \\
\bar{d}_{i} & =\overline{3}_{i}^{b} \cos \theta_{i}+\overline{3}_{i}^{c} \sin \theta_{i}, \\
L_{i} & =2_{i}^{a} \cos \theta_{i}+2_{i}^{c} \sin \theta_{i}, \\
\bar{e}_{i} & =1_{i}^{a} .
\end{aligned}
$$

(The right-handed neutrinos are identified with a linear combination $1_{i}^{b} \cos \theta_{i}+1_{i}^{c} \sin \theta_{i}$.) At this stage, there appear three degrees of freedom $\theta_{i}(i=1,2,3)$, i.e. the ways of embedding $\overline{5}_{i}$ matter in the $S U(5)$ language, which we refer to as the twisting angles in the text.

Finally we comment on the $B-L$ (baryon minus lepton number) symmetry. If one assumes that $U(1)_{B-L}$ is a gauge symmetry embedded in $E_{6}$ group, that is possible only when the matter twisting angles take the boundary values $\theta_{i}=0$ or $\pi / 2$. For $\theta_{i}=0$, the generator of $U_{B-L}$ corresponds to

$$
T_{B-L} \propto 4 T_{V}-T_{X},
$$

and for $\theta_{i}=\pi / 2$

$$
T_{B-L} \propto 16 T_{V}+T_{X}+5 T_{Z}
$$

where $T_{V}, T_{X}$ and $T_{Z}$ are the generators of $U(1)_{V}, U(1)_{X}$ and $U(1)_{Z}$. Therefore for the general matter embedding $\left(\theta_{i} \neq \theta_{j}\right)$, the $B-L$ symmetry is not local one. The continuous values of twisting angles implies that potential terms of the model should possess abelian global symmetries. One linear combination of these global and local $U(1)$ symmetries remains intact in low-energy regime as $U(1)_{B-L}$ (which is accidentally enhanced within the SM matter content).

\section{B Multiple $D$-term corrections to scalar masses}

Here we present the general parametrization of $D$-term corrections to scalar soft masses, which arise in the rank reduction of gauge symmetry [4]. As an example, let us examine the case that $E_{6}$ is broken down to $S U(5)$ as the following symmetry breaking chain:

$$
E_{6} \rightarrow S U(6) \times S U(2) \stackrel{\Lambda_{1}}{\longrightarrow} S U(5) \times U(1) \stackrel{\Lambda_{2}}{\longrightarrow} S U(5),
$$


where the breakdown occurs at two subsequent rank reduction scales $\Lambda_{1}$ and $\Lambda_{2}$. At each scale, there appears $D$-term contribution to scalar masses. For the first reduction at $\Lambda_{1}$, the correction is written as

$$
\Delta_{1} m_{i}^{2}=\left[g_{6}^{2}\left(T_{6}\right)_{i} \cos \alpha+g_{2}^{2}\left(T_{2}\right)_{i} \sin \alpha\right] D_{\Lambda_{1}}
$$

where $g_{2}$ and $g_{6}$ are the $S U(2)$ and $S U(6)$ gauge couplings, respectively, and $D_{\Lambda_{1}}$ is a mass parameter (a deviation of the symmetry-broken vacuum from supersymmetric directions). The diagonal generators for vector representation are given by $T_{6} \propto \operatorname{diag}(1,1,1,1,1,-5) \otimes 1$ and $T_{2} \propto 1 \otimes(1,-1)$, which commute with the $S U(5)$ generators. The unbroken $U(1)$ generator at $\Lambda_{1}$ is parametrized by the angle $\alpha$ as $T_{2} \cos \alpha-T_{6} \sin \alpha$, and the associated gauge coupling constant is given by

$$
\frac{1}{g_{1}^{2}}=\frac{\cos ^{2} \alpha}{g_{2}^{2}}+\frac{\sin ^{2} \alpha}{g_{6}^{2}} .
$$

At the second reduction scale $\Lambda_{2}$, there appears another $D$-term contribution due to the breaking of the above $U(1)$ symmetry:

$$
\Delta_{2} m_{i}^{2}=\left[g_{1}^{2}\left(T_{2}\right)_{i} \cos \alpha-g_{1}^{2}\left(T_{6}\right)_{i} \sin \alpha\right] D_{\Lambda_{2}}
$$

In the end, the total $D$-term correction is written as follows:

$$
\begin{aligned}
\Delta m_{i}^{2} & =\Delta_{1} m_{i}^{2}+\Delta_{2} m_{i}^{2} \\
& =\left[g_{6}^{2}\left(T_{6}\right)_{i} \cos \alpha+g_{2}^{2}\left(T_{2}\right)_{i} \sin \alpha\right] D_{\Lambda_{1}}+\left[g_{1}^{2}\left(T_{2}\right)_{i} \cos \alpha-g_{1}^{2}\left(T_{6}\right)_{i} \sin \alpha\right] D_{\Lambda_{2}}, \\
& =\left(T_{6}\right)_{i} D_{6}+\left(T_{2}\right)_{i} D_{2},
\end{aligned}
$$

where $D_{2,6}$ are the redefined mass parameters. An important fact here is that the generators $T_{6}$ and $T_{2}$ can be rewritten by suitable linear combinations of the generators of $U(1)^{2} \subset$ $E_{6} / S U(5)$. Therefore if one chooses $U(1)_{X}$ and $U(1)_{Z}$ as the basis of $U(1)^{2}$, the scalar mass correction is generically parametrized in the following form:

$$
\Delta m_{i}^{2}=\left(T_{X}\right)_{i} D_{X}+\left(T_{Z}\right)_{i} D_{Z}
$$

The parametrization is always valid for $D$-term contributions associated with $E_{6} \rightarrow S U(5)$ breaking, since any $U(1)$ generator in $E_{6} / G_{\mathrm{SM}}$ is spanned by $T_{X}$ and $T_{V}$. Thus scalar masses generally have a wide variety due to generation-dependent charges $\left(T_{X}\right)_{i}$ and $\left(T_{Z}\right)_{i}$, according to the matter embedding discussed in Appendix $\mathrm{A}$. 
The result is easily generalized to the case with an arbitrary gauge group $G_{U}$ which is a rank $n$ semi-simple group. Above the unification scale, $G_{U}$ is broken down to $S U(5)$ and the $D$-term contributions generally appear. One is always able to take a linearly independent set of $U(1)$ generators $T_{\ell}(\ell=1, \cdots, n-4)$ as the basis of Cartan generators of $G_{U} / S U(5)$. Using the generators $T_{\ell}$, the general form of scalar mass corrections is written as follows:

$$
\Delta m_{i}^{2}=\sum_{\ell=1}^{n-4}\left(T_{\ell}\right)_{i} D_{\ell} .
$$

The parametrization is valid for arbitrary symmetry breaking chains. If the charges $\left(T_{\ell}\right)_{i}$ are generation dependent according to the matter assignment in unified theory, generationdependent contributions to scalar masses arise from the $D$ terms. 


\section{References}

[1] J.R. Ellis, S. Kelley and D.V. Nanopoulos, Phys. Lett. B260 (1991) 131; U. Amaldi, W. de Boer and H. Furstenau, Phys. Lett. B260 (1991) 447; P. Langacker and M.x. Luo, Phys. Rev. D44 (1991) 817.

[2] H.P. Nilles, Phys. Rept. 110 (1984) 1; S.P. Martin, arXiv:hep-ph/9709356.

[3] N. Polonsky and A. Pomarol, Phys. Rev. D51 (1995) 6532.

[4] M. Drees, Phys. Lett. B181 (1986) 279; J.S. Hagelin and S. Kelley, Nucl. Phys. B342 (1990) 95; Y. Kawamura, H. Murayama and M. Yamaguchi, Phys. Lett. B324 (1994) 52; Phys. Rev. D51 (1995) 1337; H.C. Cheng and L.J. Hall, Phys. Rev. D51 (1995) 5289; C.F. Kolda and S.P. Martin, Phys. Rev. D53 (1996) 3871.

[5] R. Rattazzi and U. Sarid, Phys. Rev. D53 (1996) 1553; T. Blazek, R. Dermisek and S. Raby, Phys. Rev. D65 (2002) 115004; D. Auto, H. Baer, C. Balazs, A. Belyaev, J. Ferrandis and X. Tata, JHEP 0306 (2003) 023; K. Inoue, K. Kojima and K. Yoshioka, JHEP 0607 (2006) 032; Phys. Lett. B644 (2007) 172.

[6] H. Georgi and S.L. Glashow, Phys. Rev. Lett. 32 (1974) 438.

[7] M. Maltoni, T. Schwetz, M.A. Tortola and J.W.F. Valle, New J. Phys. 6 (2004) 122; A. Bandyopadhyay, S. Choubey, S. Goswami, S.T. Petcov and D.P. Roy, Phys. Lett. B608 (2005) 115; G.L. Fogli, E. Lisi, A. Marrone and A. Palazzo, Prog. Part. Nucl. Phys. 57 (2006) 742.

[8] F. Gursey, P. Ramond and P. Sikivie, Phys. Lett. B60 (1976) 177; Y. Achiman and B. Stech, Phys. Lett. B77 (1978) 389; R. Barbieri and D.V. Nanopoulos, Phys. Lett. B91 (1980) 369.

[9] J. Sato and T. Yanagida, Phys. Lett. B430 (1998) 127; Y. Nomura and T. Yanagida, Phys. Rev. D59 (1999) 017303; M. Bando and T. Kugo, Prog. Theor. Phys. 101 (1999) 1313; ibid. 104 (2000) 211; Phys. Lett. B483 (2000) 163; M. Bando and N. Maekawa, Prog. Theor. Phys. 106 (2001) 1255.

[10] R. Slansky, Phys. Rept. 79 (1981) 1; Y. Kawamura and M. Tanaka, Prog. Theor. Phys. 91 (1994) 949; ibid. 93 (1995) 789. 
[11] H. Nakano, arXiv:hep-th/9404033; E. Dudas, S. Pokorski and C. A. Savoy, Phys. Lett. B369 (1996) 255; G.R. Dvali and A. Pomarol, Phys. Rev. Lett. 77 (1996) 3728; T. Kobayashi, H. Nakano, H. Terao and K. Yoshioka, Prog. Theor. Phys. 110 (2003) 247; K.S. Babu, T. Enkhbat and I. Gogoladze, Nucl. Phys. B678 (2004) 233.

[12] F. Gabbiani, E. Gabrielli, A. Masiero and L. Silvestrini, Nucl. Phys. B477 (1996) 321.

[13] Z. Maki, M. Nakagawa and S. Sakata, Prog. Theor. Phys. 28 (1962) 870.

[14] A.Y. Smirnov, Phys. Rev. D48 (1993) 3264; M. Tanimoto, Phys. Lett. B345 (1995) 477; M. Bando, T. Kugo and K. Yoshioka, Phys. Rev. Lett. 80 (1998) 3004; G. Altarelli, F. Feruglio and I. Masina, Phys. Lett. B472 (2000) 382.

[15] F. Borzumati and A. Masiero, Phys. Rev. Lett. 57 (1986) 961; R. Barbieri, L.J. Hall and A. Strumia, Nucl. Phys. B445 (1995) 219; J. Hisano, T. Moroi, K. Tobe and M. Yamaguchi, Phys. Rev. D53 (1996) 2442.

[16] W.M. Yao et al. [Particle Data Group], J. Phys. G 33 (2006) 1.

[17] K. Choi, K.S. Jeong and K.i. Okumura, JHEP 0509 (2005) 039; M. Endo, M. Yamaguchi and K. Yoshioka, Phys. Rev. D72 (2005) 015004. 